\title{
Nanofiber containing carbon nanotubes enhanced PC12 cell proliferation and neuritogenesis by electrical stimulation
}

\author{
Wen-Ta Su ${ }^{*}$ and Yi-An Shih \\ Department of Chemical Engineering and Biotechnology, National Taipei University of Technology, 1 \\ Sec. 3, Chung-Hsiao E. Rd, Taipei 10608, Taiwan
}

\begin{abstract}
The nervous system is an important regulator of the human body because it adapts our responses to the external environment and provides people the ability of thought, memory, and emotion. PC12 is a cell line that is commonly used to study the behavior of neural differentiation. PC12 cells further differentiate into nerve cells when stimulated by nerve growth factor (NGF), which have neurite, dendrite, and axon, and form synapses with neighboring cells to build neural networks. Micropatterns and electric stimulation can significantly influence cellular attachment, proliferation, orientation, extracellular matrix (ECM) expression, neural differentiation, and cellular motion. We fabricated polycaprolactone (PCL) nanofiber with or without carbon nanotubes (CNTs) by electrospinning and promoted the neural differentiation of PC12 cells by electric stimulation. We used scanning electron microscope (SEM) and fluorescence microscope to observe the NGF-induced growth of PC12 cells on PCL nanofiber. Axon formation and cellular activity expression, that confirm that PC12 cells can grow well on PCL nanofiber, and the gene expressions of MAP1b and GAP43 significantly increased after electric stimulation. Based on the results, the structure of nanofibers containing CNTs can effectively induce neural differentiation of PC12 cells in an electric field. This experimental model can be used for future clinical applications.
\end{abstract}

Keywords: PC12 cells, carbon nanotube, electric stimulation, neuritogenesis

\section{Introduction}

Tissue engineering for reconstructive surgery requires some important factors including suitable cellular sources, optimal signals, and a biodegradable scaffold to serve as a temporary to provide cellular growth and regulate intercellular communication [1]. Nanofibers that are composed of ultrafine and continuous fibers recently attracted attention in regenerative medicine because of their high ratio of the surface to volume, higher porosity, and a capacity to mimic the natural ECM structure for tissue engineering. Electrospinning uses a high electric field to draw the polymer solution into continuous fibers and can fabricate different fibrous scaffolds that imitate the microstructure of the natural ECM [2].

Regeneration of injured nerve is achieved by chemical and physical treatments. Chemical treatments

\footnotetext{
* Address for correspondence: Wen-Ta Su, Department of Chemical Engineering and Biotechnology, National Taipei University of Technology, 1 Sec. 3, Chung-Hsiao E. Rd, Taipei 10608, Taiwan. Tel.: +886-2-27712171 (ext. 2554); Fax: +886-2-27519204; E-mail: f10549@ntut.edu.tw.
} 
include some neurotrophic factors that can enhance effectively peripheral nerve regeneration in the initial stage, whereas physical treatments such as electrical stimulation recover the function of injured nerve in the neural system. Previous studies indicated that electric stimulation can enhance neurite outgrowth in vivo and in vitro [3].

Inducing neurite outgrowth is required in neural growth processes during neural regeneration and development. Neural cells growth is affected by environmental interactions, such as electrical difference, chemical difference, ECM construction, mechanical strength, and contact guidance of surface characteristics. These environmental cues can affect the neural growth cones (located at motile tips of neurites), and then transduced cellular growth signals toward proper targets. In additional, studying nervous system can also help the designing of parameters for neural implantable that can orientate correctly neurite outgrowth in vivo.

Human nerve cells possess low proliferated capacity and differentiation rates. So the neural cell lines normally add growth factors, like brain derived neurotrophic factor (BDNF), nerve growth factor (NGF), neurotropins-3, and retinoic acid (RA) for cellular growth well in vitro. PC12 rat pheochromocytoma cells are widely used in investigating neural behavior because of they would express some neural characteristics reversibly when expose to growth factor supplement. On the whole, PC12 cells grow in the culture medium containing $50 \mathrm{ng} / \mathrm{mL}$ NGF resulted the mitosis and spread the cellular structure that are morphological correspondent with primary sympathetic neurons [4].

\section{Materials and methods}

\subsection{Preparation of PCL nanofibers containing carbon nanotubes}

Carbon nanotubes (CNTs, Centron Biochemistry Technology Co., Ltd., Taiwan) was fully rinsed with acid solution and then dried in the oven. Polycaprolactone (PCL) nanofibers were fabricated using an electrospinning system composed of a syringe pump, a syringe, Teflon fluidic tubing, an 18-gauge blunt-tip catheter, and an adapter. A high-voltage power source connected a standard alligator clamp with a catheter tip, and the collector is a piece of aluminum foil with electrical tape. Then, 14\% PCL ( $80000 \mathrm{Mw}$, Sigma-Aldrich Corporation, USA) solution with or without $0.1 \%$ CNTs were placed in a $10 \mathrm{~mL}$ syringe connected to an 18-gauge needle with a tip-to-collector distance of 23 $\mathrm{cm}$. The solution was fed into the needle by a syringe pump at a rate of $2 \mathrm{~mL} / \mathrm{h}$, and a voltage of $18 \mathrm{kV}$ was applied to generate a polymer jet.

\subsection{Cell culture and cell proliferation assay}

PC12 cell line was bought from Bioresource Collection and Research Center (Taiwan), and then cells seeded in DMEM medium containing 10\% horse serum (Sigma) and 5\% fetal bovine serum (Sigma) for routine culture under an atmosphere of $5 \% \mathrm{CO}_{2}$ incubator at $37^{\circ} \mathrm{C}$. The cultivated medium was exchanged every $2 \mathrm{~d}$ to $3 \mathrm{~d}$. The cellular density of $1 \times 10^{5}$ cells $/ \mathrm{mL}$ was seeded on the PCL nanofibers for 1 day culture, then the cultivated medium was changed into $50 \mathrm{ng} / \mathrm{mL}$ NGF and $2 \%$ FBS mixture to influence cellular differentiation and promote the neurite formation. The cultivating devices operated under $750 \mathrm{mV}$ and $100 \mathrm{~Hz}$ alternating current stimulation for 30 min daily for 3 and $6 \mathrm{~d}$. The cell viability on cultivated substrate were measured using commercially available 3-(4, 5-dimethylthiazol-2-yl)-2, 5-diphenyltetrazolium bromide (MTT, Sigma). The metabolized results were measured by a spectrophotometer (Multiskan ${ }^{\mathrm{TM}}$ FC, Thermo Scientific ${ }^{\mathrm{TM}}$, USA) at $540 \mathrm{~nm}$. 


\subsection{Cellular morphology observation}

Passed through various incubation periods, PC12 cells grown on pure PCL nanofibers were fixed with $4 \%$ paraformaldehyde solution at $4^{\circ} \mathrm{C}$ for $1 \mathrm{~h}$. After fixation, all samples were washed twice in $5 \%$ sucrose/PBS solution for $15 \mathrm{~min}$, dehydrated in sequentially increasing ethanol concentractions to $100 \mathrm{vol} \%, 30 \mathrm{~min}$ in each steps and then dried in a CO2 critical point dryer (Pelco CPD\#2400) for ethanol removed fully. Finally, the samples were sputter-coated with a slight thick gold layer by a sputter coater (Cressington 108 manual) and observed by a SEM (FEI Quanta 200, Philips, Netherlands). In immunostaining process, all specimens were rinsed twice in PBS to remove all suspended cells. Cultivated cells fixed in $4 \%$ paraformaldehyde for $20 \mathrm{~min}$ and permeabilized in $1 \%$ Triton X100. Filamentous actin was stained by phalloidin-TRITC (Sigma), whereas the nucleus was stained with DAPI (Molecular Probes). All samples were observed by a confocal laser scanning microscope (TCS-AOBS-SP5; Axiovision software).

\subsection{Gene expression by RT-PCR}

The total RNA extracted from cultivated cells by Trizol reagent (Ambion ${ }^{\circledR}$, Life Technologies ${ }^{\mathrm{TM}}$, USA) and quantitated by a spectrophotometer at $260 \mathrm{~nm} / 280 \mathrm{~nm}$ absorbance ratio. Taking $500 \mathrm{ng}$ of the RNA was reverse transcribed to cDNA using Super Script ${ }^{\circledR}$ III Reverse Transcriptase kit (Life Technologies ${ }^{\mathrm{TM}}$, USA), and real-time PCRs operated according to Invitrogen ${ }^{\mathrm{TM}}$ Corporation (USA) methods. Reverse transcription PCR (RT-PCR) was measured by Smart Quant Green Master Mix with dUTP and ROX following to Protech Technology Enterprise Co., Ltd. (Taiwan) methods. A forty-five cycles performed to analyze the target genes and GAPDH gene. The sequences of primers including microtubule-associated protein 1(MAP1b), growth-associated protein 43 (GAP43), neurofilament-L (NF-L), and GAPDH were shown in Table 1.

\subsection{Statistical analysis}

Data are presented as means \pm standard deviation (SD), and statistical analysis was performed by analysis of variance. Student's t-test was performed, and $\mathrm{P}$ values smaller than 0.05 represents significant differences vs control group.

Table 1

Sequences of used primers for the RT-PCR

\begin{tabular}{|l|l|}
\hline Gene name & primer sequences \\
\hline MAPb1 & Forward: AAGCTGAGAGGTCCCTTATGTCC \\
\hline & Reverse: AATGAGCTCCAGCTGAGGCTTG \\
\hline GAP43 & Forward: \\
& AGAACATAGAAGCTGTAGATGAAAC \\
\hline & Reverse: CCATTTCTTAGAGTTCAGGCAT \\
\hline NF-L & Forward: \\
& AATAAGTCGACGCTGCAGGACCTCAACCA \\
\hline & Reverse: \\
& GATCTGAATTCCTGAGCCTGGTCTCTTC \\
\hline GAPDH & Forward: ATGAGAAGTATGACAACAGCC \\
\hline & Reverse: AGTCCTTCCACGATACCAA \\
\hline
\end{tabular}



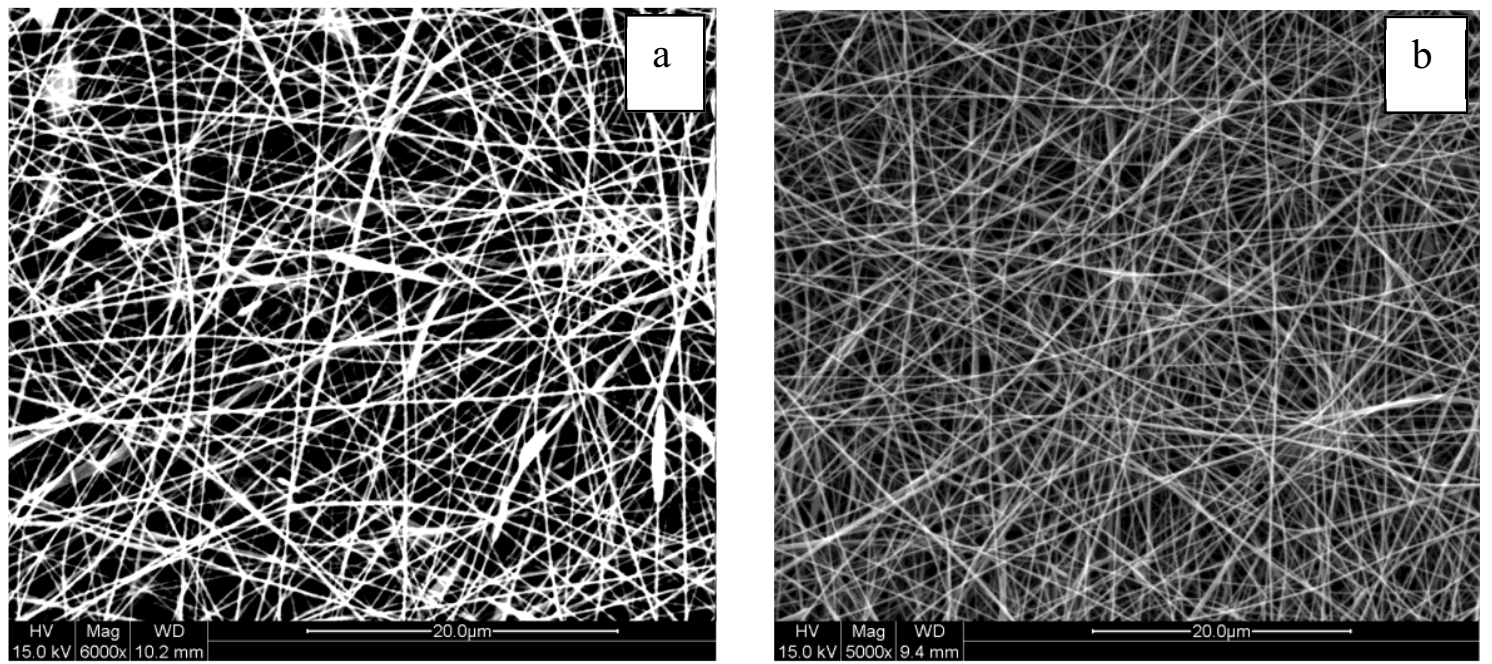

Fig. 1. The morphology of $14 \%$ PCL nanofibers (a) and 14\% PCL nanofibers containing $0.1 \%$ CNTs (b).

\section{Results and discussion}

\subsection{Material character of PCL nanofibers with and without carbon nanotubes}

The images of outside morphology and microstructure of cross section for the used PCL nanofibers were shown in Figure 1. All fabricated fibers exhibited a 3D, porous, and fibrous structure with interconnected pores and randomly oriented fibers. The morphology of PCL/CNT-blended nanofibers was similar to that of pure PCL nanofibers. The surface wettability of the electrospun PCL with and without CNTs was assayed. The contact angles of pure PCL and PCL/CNT-blended fibers were measured to be $128.71^{\circ}$ and $43.86^{\circ}$, respectively, indicating that pure PCL fibers are extremely hydrophobic, whereas PCL/CNT-blended fibers are hydrophilic. Thus, adding CNTs can decrease the contact angle and increase the hydrophilic property of PCL fibers for cellular attachment.

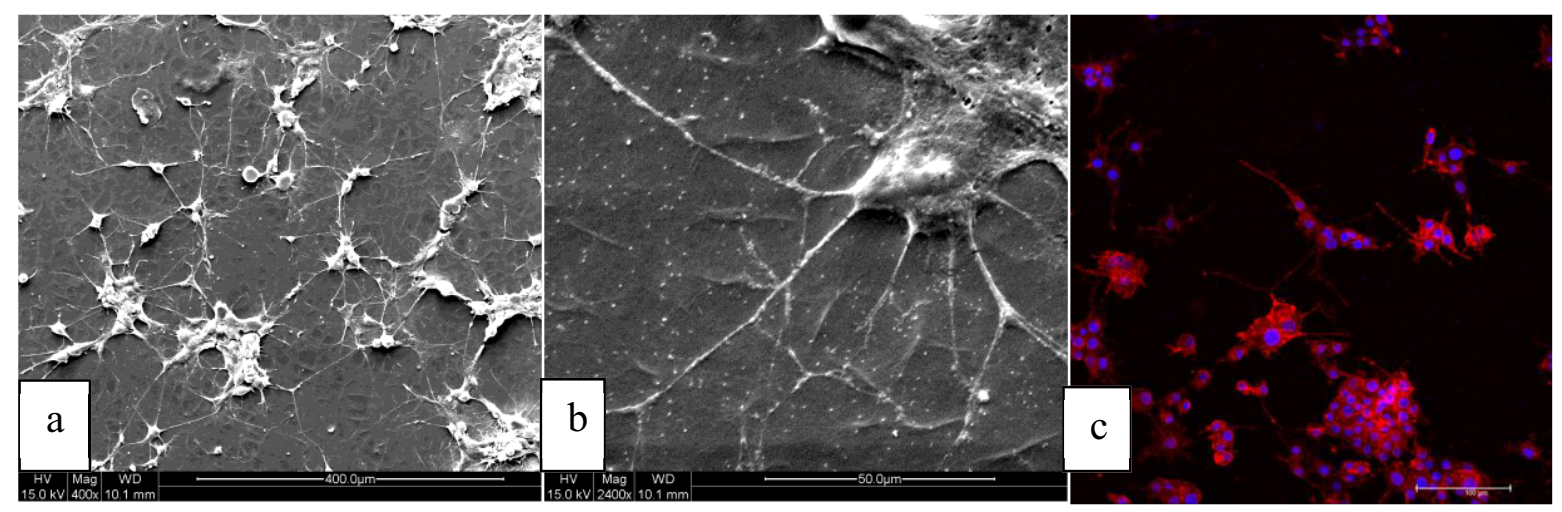

Fig. 2. The SEM image of cellular morphology of induced PC12 cells on pure PCL nanofiber by 50 ng/L NGF for 6 days culture $(a, b)$, and the image of fluorescence immunostaining, blue indicates the nucleus staining and actin is labeled in red (c). The scale bars are $400 \mu \mathrm{m}$ (a), $50 \mu \mathrm{m}$ (b) and $100 \mu \mathrm{m}$ (c). 


\subsection{Morphology and proliferation of PC12 cells}

NGF can stimulate the morphological changing of PC12 cells similar to neural cells appeared the neurite outgrowth and elongation. The SEM images show that PC12 cells were successfully seeded and grown on the fabricated nanofibers. PC12 cells attached initially, spread rapidly on PCL nanofibers, and then formed neurites on the tested fibers (Figure 2). In addition, the majority of cellular neurites formed on fibers were observed after $6 \mathrm{~d}$ of NGF-inducing culture. Neurite formations observed and imaged by SEM (Figure 2(b)) and a fluorescence microscope that is an inverted microscope with DIC optics (Figure 2(c)). Results suggest that the PCL fibers are suitable for PC12 cell attachment, spreading, growth, and differentiation.

The proliferation of PC12 cells growth on the nanofibrous scaffolds were measured by MTT assay. The result of MTT was directly proportional to the number of viable cells present. Cellular proliferation on pure PCL and PCL/CNT-blended nanofibers with or without electric stimulation process after $3 \mathrm{~d}$ of culture is shown in Figure 3. The cellular numbers on pure PCL nanofibers were lower than those on PCL/CNT-blended nanofibers during cellular cultivation. However, the proliferated cell numbers of both nanofibers under electric stimulation process did not show distinct difference. Thus, electric stimulation process was not helpful in PC12 cell proliferation.

\subsection{Neuritogenesis analysis of PC12 cells}

The results from the RT-PCR indicated that nerve-related genes can be detected in this inducing culture system. Figure 4 shows the gene expression quantified in expressed folds than the control gene in nanofibers for 3 and $6 \mathrm{~d}$ of culturing period. PC12 cells with alternating current stimulation have lower MAP1b, GAP43, and NF-L expressions than those cells cultured on nanofibers without electric simulation (Figure 4(a)), thereby indicating that electric field has no inducing effect on pure PCL fiber. PC12 cells have higher MAP1b and GAP43 expressions on PCL/CNT-blended nanofibers under

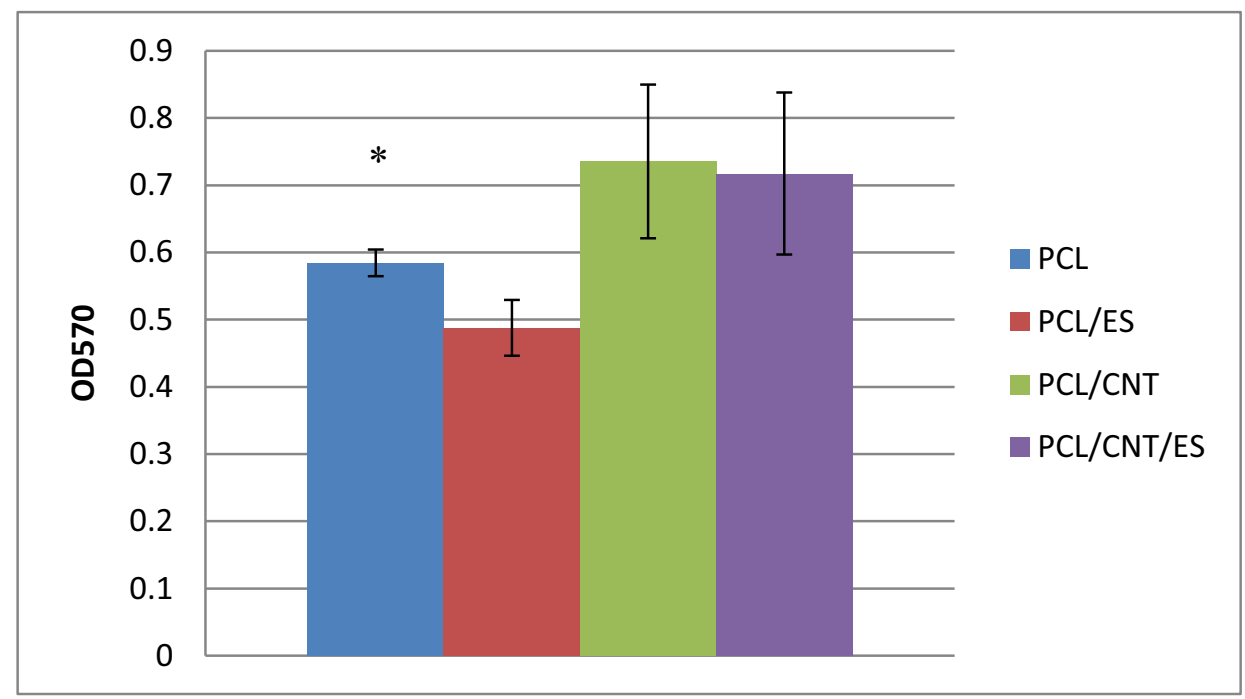

Fig. 3. Cellular numbers were examined by MTT. PCL is $14 \%$ PCL, PCL/ES is $14 \%$ PCL under electrical stimulation $\mathrm{PCL} / \mathrm{CNTs}$ is $14 \% \mathrm{PCL}$ added $0.1 \% \mathrm{CNTs}$ and PCL/CNTs/ES is $14 \%$ PCLadded $0.1 \%$ CNTs under electrical stimulation. Data are plotted as mean \pm standard deviation $(n=3), *, p<0.05$ vs control group. 

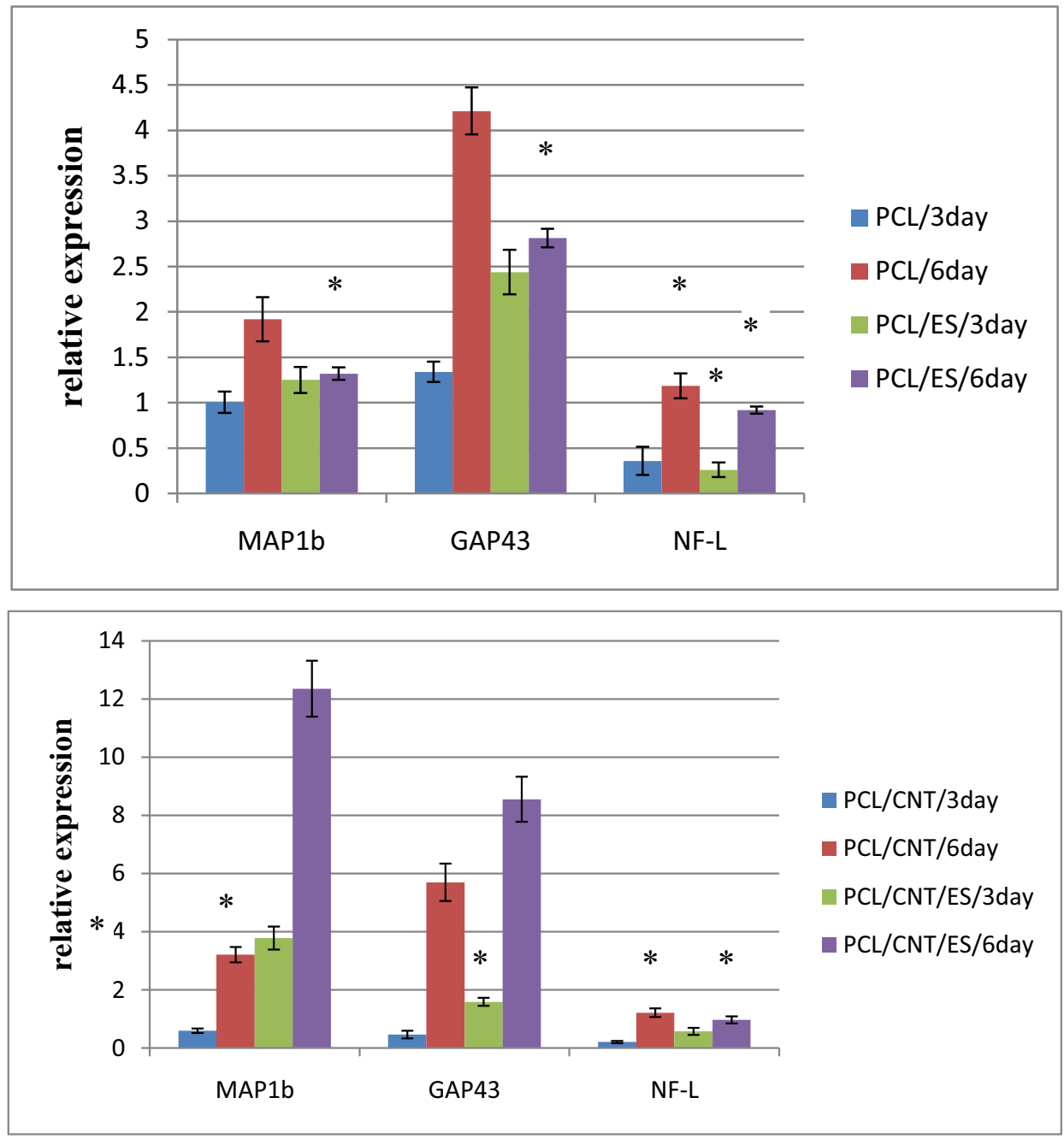

Fig. 4. Gene expression of PC12 cells cultivated on PCL nanofibers without CNTs (a) and PCL nanofibers containing CNTs (b) on ES process. Data are plotted as mean \pm standard deviation $(n=3), *, p<0.05$ vs control group.

electric stimulation (Figure 4(b)), but NF-L expression was similar on PCL nanofibers with or without CNTs. CNTs have electric conductivity that simulates cellular differentiation by electric field, as indicated in Figure 4(b). RT-PCR data confirm that the genetic differentiation of PC12 cells was superior in PCL nanofibers with CNTs than in pure PCL nanofibers under electric simulation.

\section{Conclusions}

This study confirmed that CNTs can induce neural differentiation of PC12 cells under electric simulation. Results show that cellular functions are affected by PCL nanofibers containing CNTs. CNT-induced PC12 cells appeared at fast growth rate, and had the maximum MAP1b, GAP-43, and NF-L expressions in an electric field. To investigate different cellular behaviors is a useful method for 
designing and developing strategies for tissue engineering.

\section{References}

[1] W.T. Su and X.W. Chen, Stem cells from human exfoliated deciduous teeth differentiate into functional hepatocyte-like cells by herbal medicine, Bio-Medical Materials and Engineering 24 (2014), 2243-2247.

[2] W.T. Su, P.S. Wu and T.Y. Huang, Osteogenic differentiation of stem cells from human exfoliated deciduous teeth on poly (E-caprolactone) nanofibers containing strontium phosphate, Materials Science and Engineering: C 46 (2015) 427-434.

[3] X. Liu, K.J. Gilmore, S.E. Moulton and G.G. Wallace, Electrical stimulation promotes nerve cell differentiation on polypyrrole/poly (2-methoxy-5 aniline sulfonic acid) composites, Journal of Neural Engineering 6 (2009), 065002.

[4] J.D. Foley, E.W. Grunwald, P.F. Nealey and C.J. Murphy, Cooperative modulation of neuritogenesis by PC12 cells by topography and nerve growth factor, Biomaterials 26 (2005), 3639-3644. 